\title{
EL USO DE MUNDOS VIRTUALES INMERSIVOS EN LA MEJORA TRANSVERSAL DE COMPETENCIAS DE TRABAJO EN EQUIPO, CREATIVIDAD E INNOVACIÓN
}

\section{THE USE OF IMMERSIVE VIRTUAL WORLDS IN THE IMPROVEMENT OF COMPETENCIES OF TEAM WORK, CREATIVITY AND INNOVATION}

\author{
Leovigilda Ortiz-Medina(*) \\ Elvira Fernández-Ahumada \\ Encarnación V. Taguas \\ José Antonio Adame \\ Pablo Lara \\ Eduardo Zamora \\ José Emilio Guerrero \\ pa2ormel@uco.es (Departamento de Producción Animal, Campus_Rabanales)
}

University of Cordoba

\begin{abstract}
The Teaching Innovation Plan 2015-2016 of the University of Cordoba had among its priorities competency-based work (line 1), the promotion of virtual teaching (line 10) and tutoring students (line 12). In this context, the use of immersive virtual worlds has been applied to improve teamwork competencies and the management of virtual tools, both being important and transferable aspects to the learning of any discipline. Thus, an immersive virtual scenario was created to enable work meetings, and group activities were designed for learning the handling of avatars in working groups. In addition, tutored activities aimed at solving group doubts as well as definitely improving creativity in an innovative and different atmosphere. These sessions were included within the practices of the subject "Projects" of the degree of Agri-food Engineering and Rural Environment. The results of the evaluation of the experience -as an ad hoc survey designed to characterize the learning curve of the management of avatars and immersive worlds-, showed the success and usefulness of the project whose material is reusable in future educational experiences.Keywords: avatar; immersive worlds; teamwork; virtual teaching.
\end{abstract}

\section{Resumen}

El Plan de Innovación Docente 2015-2016 de la Universidad de Córdoba tenía entre sus líneas prioritarias tanto el trabajo en competencias (línea 1), como el fomento de la enseñanza virtual (línea 10) y la tutorización del alumnado (línea 12). En este contexto, nuestro trabajo ha aplicado y evaluado el uso de mundos virtuales inmersivos para la mejora del manejo de herramientas virtuales y competencias de trabajo en equipo, aspectos claves y transferibles al aprendizaje de cualquier disciplina. Así, se creó un escenario virtual inmersivo para reuniones de trabajo y se diseñaron unas actividades grupales que incluían un manual para el aprendizaje del manejo de avatares y su interacción en un grupo de trabajo en mundos inmersivos. Además se llevaron a cabo actividades tutoradas dirigidas a resolver dudas en grupo así como para definitivamente mejorar la creatividad en un contexto innovador y diferente. Estas sesiones se incluyeron dentro de las prácticas de la asignatura "Proyectos" del grado de Ingeniería Agroalimentaria y del Medio Rural. Los resultados de la evaluación de la experiencia a modo de encuestas diseñadas ad hoc para caracterizar la curva de aprendizaje del manejo de avatares y mundos inmersivos, puso de manifiesto el éxito y utilidad del proyecto cuyo material es reutilizable en futuras experiencias educativas.

Palabras clave: avatar, mundos inmersivos, equipos de trabajo, enseñanza virtual.

\section{INTRODUCCIÓN}

Cada vez con mayor frecuencia se está asociando la calidad en la enseñanza con la mejora de la inserción laboral de los egresados. Distintos estudios realizados indican la necesidad de proporcionar a los estudiantes una amplia gama de habilidades y conocimientos más allá de los meramente técnicos (Ohland et al., 2004), incluyendo habilidades de comunicación, capacidad de trabajo en equipos multidisciplinares, espíritu emprendedor, enfoques globales y multilaterales para la resolución de problemas, así como la obligada sensibilidad por el entorno cultural, social y económico.

La difusión de procesos de trabajo flexibles (como la rotación del personal en las tareas productivas; el incremento en la responsabilidad y la conformación y desarrollo de equipos de trabajo; la subcontratación; el aumento en la capacitación y entrenamiento laboral, etc.,) es uno de los ejes mediante el cual se está impulsando el incremento en la productividad y la mejora en la competitividad en las organizaciones e implica una nueva forma de entender la organización del trabajo. Desde esta perspectiva los sistemas de trabajo en equipo están adquiriendo una importancia fundamental, no sólo para la mejora de la competitividad sino como un sistema de organización del trabajo que permite mejorar el clima laboral, la comunicación interna de los nuevos miembros, la transmisión de sus valores y cultura, etc. (Palomo Vadillo, 2013). 
La inclusión del trabajo en equipo como metodología docente para la formación en competencias ha sido evaluada en distintas asignaturas, especialmente en la asignatura de Taller de Empresas de la Universidad de Córdoba, por parte de los docentes implicados y mediante procesos de evaluación realizados con los estudiantes, quedando patente la dificultad de esta práctica, y la necesidad de monitorización del trabajo en equipo para la obtención de los deseados resultados de aprendizaje, evitando las disfuncionalidades en los grupos. Uno de los principales problemas citados por los alumnos es la dificultad de mantener reuniones de trabajo eficaces (limitación de tiempo y de horarios compatibles, dificultades para disponer de lugares de encuentro adecuados, etc.).

Una de las formas con las que se están solventando las cuestiones logísticas de las reuniones de equipos de trabajo, en la práctica empresarial cotidiana, es mediante el uso de las nuevas tecnologías de la comunicación ya que, cada vez con más frecuencia, estos equipos se constituyen por trabajadores que se encuentran en localizaciones geográficas distintas. Un avance en los sistemas de telecomunicación lo constituyen los entornos virtuales en plataformas Web. La realidad virtual y los mundos inmersivos (Virtual Environments) se refieren a ambientes artificialmente generados por ordenador donde los usuarios interactúan a través de un individuo figurativo que los representa en dicho ambiente (su "avatar"). Así, a partir de un software de dominio multiusuario, cada participante está presente en un entorno tridimensional a través de su representación gráfica, "avatar", que interactúa y emula un situación real (Baker et al., 2009). Autores como Schmorrow (2009) sugieren que los ambientes virtuales serán la tecnología de principios del siglo XXI que cambiará más dramáticamente el modo en que vivimos, particularmente en las áreas educativas, de desarrollo de productos y de entretenimiento. El éxito de los ambientes virtuales está bien documentado en diversos campos como medicina (Gerald y Antonacci, 2007), industria (Wilson, 2008), y fundamentalmente en educación (Bouras y Tsiatsos, 2006; Sumners y Reiff, 2008; Lorenzo et al., 2012; Mathers et al., 2012), entre muchos otros. Se puede citar, en particular el trabajo de Mamo et al. (2011) quien destacó el éxito didáctico de un ambiente virtual para actividades de ciencias del suelo con avatares, que fue utilizado por un grupo de 126 estudiantes de la Universidad de Nebraska en EE.UU.

\section{OBJETIVOS}

El objetivo principal de este trabajo fue la creación/adaptación de un espacio virtual inmersivo y el diseño de unas actividades grupales para mejorar las competencias vinculadas al trabajo en equipo y la mejora de la creatividad en un contexto innovador y diferente. Este objetivo principal se dividió en los siguientes objetivos específicos:

1) Diseño y ejecución de un espacio inmersivo y sesiones de trabajo para el seguimiento y monitorización de reuniones de grupos de trabajo orientados a trabajo en proyectos;

2) Exploración de los mundos inmersivos para la mejora de la interacción de los estudiantes en equipos de trabajo.

\section{Metodología}

La experiencia se organizó en tres paquetes de trabajo o capítulos:

1) Diseño y adaptación del espacio virtual para las reuniones de equipos de trabajo y tutorías virtuales (Objetivo 1); Se utilizó la herramienta OPEN SIM para la construcción de escenarios adecuados a reuniones de equipo de distinto tamaño y con distintas necesidades; uso de multimedia, modelos, etc. La asesora técnica externa Carmen Beato se encargó de acondicionar el espacio virtual original.

2) Actividades de preparación de material y monitorización de las reuniones de trabajo (Objetivo 1). Se preparó el material descrito en la Figura 1 y se realizaron varias sesiones de trabajo en equipo y tutorías dirigidas donde participaron de forma colectiva e individual profesores y alumnos (Figura 2)
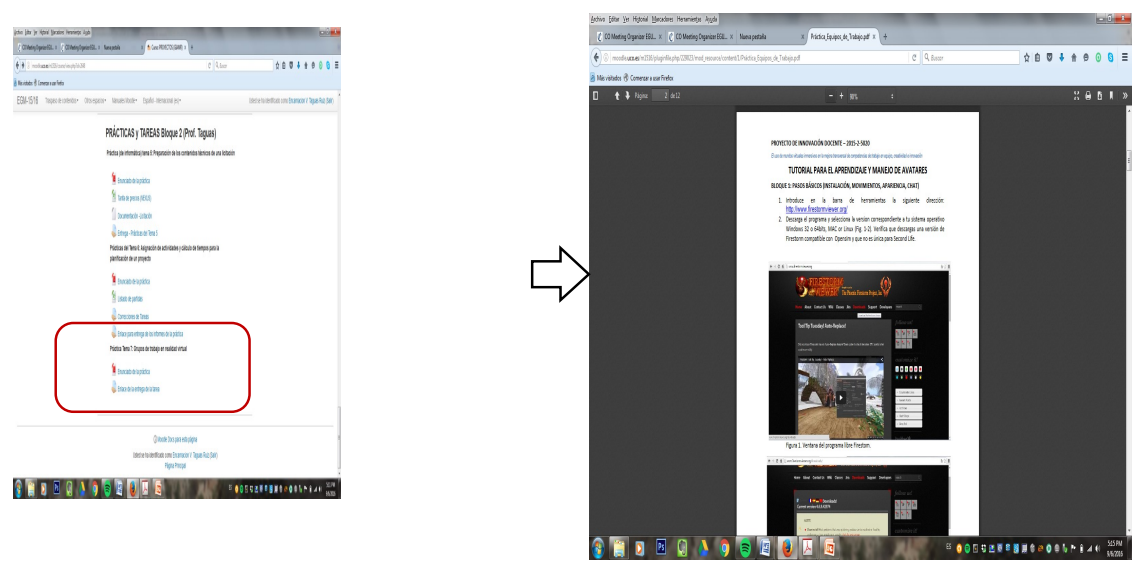
Figura 1.

Material de prácticas para la realización de las sesiones grupales en mundo virtual inmersivo: a la izquierda, enlace a Moodle y a continuación la primera página del manual.

3) Medidas del impacto de la experiencia a partir de cuestionarios y testimonios de los participantes (Objetivo 2). Se recogió a través de una encuesta información relativa a la curva de aprendizaje, la organización, ejecución y resultados de la reunión. Asimismo, se recopilaron comentarios de la experiencia de los participantes.

\section{DESCRIPCIÓN}

Se desarrolló y adaptó un espacio virtual para "reuniones de equipos de trabajo" que se llevaron a cabo para la realización de consultas y tutorías de la asignatura de Proyectos del grado de Ingeniería Agrolimentaria y del Medio Rural.. Una vez preparada la plataforma virtual con apoyo técnico externo se procedió a la realización de las actividades previstas. De esta manera los estudiantes en una primera fase, se encargaron de familiarizarse con las características del mundo virtual y el uso de avatares a partir del material preparado por profesores participantes dispuesto en Moodle (Figura 1) y en segunda instancia se realizaron reuniones de trabajo grupal y consulta (Figura 2). El uso de "avatares" facilitó la interacción por su carácter lúdico y distendido, comúnmente asociado a videojuegos o a animación. Finalmente para evaluar el impacto se preparó y realizó una encuesta con el objetivo de valorar la curva de aprendizaje y el resultado de la experiencia.
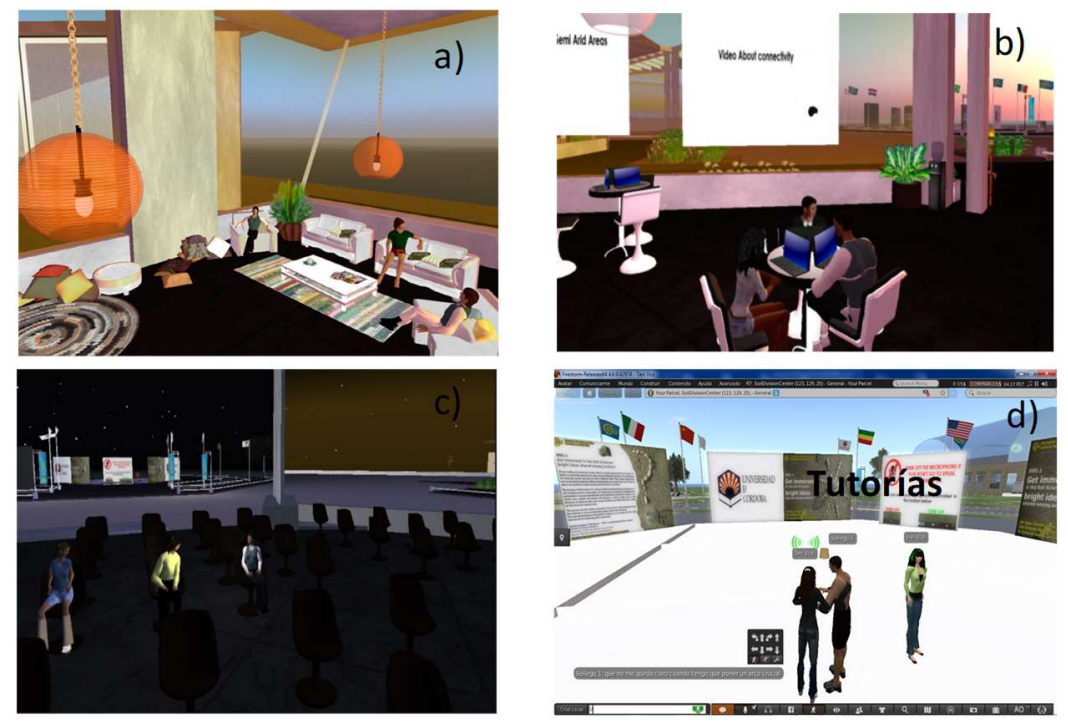

Figura 2 .

(a), (b) y (c) muestran reuniones virtuales recopiladas por los estudiantes mientras que en (d) se muestra una tutoría virtual.

\section{RESULTADOS OBTENIDOS}

En relación a los resultados obtenidos se destacan dos apartados:

i) Impacto y caracterización de curvas de aprendizaje para el manejo de los mundos virtuales inmersivos;

ii) Material creado.

Respecto a la evaluación y análisis de impacto (i), en la Tabla 1, se presenta la caracterización inicial de los estudiantes participantes. La encuesta se realizó con carácter voluntario, obteniéndose un total de 22 respuestas, lo que representa el $31 \%$ de los 70 alumnos matriculados en la asignatura. 
Tabla 1. Estadísticos básicos obtenidos de las encuestas realizadas para evaluar el impacto del proyecto.

\begin{tabular}{cccc}
\hline Análisis preliminar $(\mathbf{n}=\mathbf{2 2})$ & Característica & Media & Desv. \\
\hline $\begin{array}{c}\text { CARACTERIZACIÓN PREVIA } \\
\text { DE LOS PARTICIPANTES }\end{array}$ & Edad & 22.5 & 2.3 \\
& Sexo & $50 \% \mathrm{H} / \mathrm{M}$ & - \\
& Experiencia previa & $50 \%$ Nula; $32 \%$ Baja; & - \\
& & $9 \%$ Media $/$ alta & \\
\hline AUTOEVALUACIÓN & Ofimática & 3.2 & 1.1 \\
& e-learning & 3.8 & 1.0 \\
& Redes sociales & 3.9 & 1.1 \\
& Videojuegos & 3.1 & 1.5 \\
& Mundos inmersivos & 1.9 & 1.0 \\
\hline
\end{tabular}

Como puede observarse en la Tabla 1 se trata de estudiantes entre 22 y 23 años con poca experiencia en el manejo de mundos inmersivos y plataformas virtuales. En la Tabla 2 se muestran los resultados de las encuestas donde hay que destacar las evaluaciones positivas obtenidos sobre el diseño y estilo del entorno y sus utilidades (3.6 sobre 5) así como el apoyo prestado por el manual (3.9 sobre y 5) y de las sesiones tutoradas por el profesor (3.8 sobre 5). La presencia de los compañeros dentro del mundo virtual fue mejor valorada que la del profesor y la sensación de inmersión obtenida tuvo una puntuación notable (3.4 sobre 5). La curva de aprendizaje media mostró que el tiempo medio para un manejo básico del avatar fue alrededor de media hora (Tabla 2).

Tabla 2. Resumen de las encuestas de evaluación del entorno, competencia básica de manejo, interacción y tiempos de aprendizaje.

\begin{tabular}{|c|c|c|c|}
\hline Análisis & Ítems & Media & Desv. \\
\hline \multirow[t]{3}{*}{ ENTORNO (1-5) } & Espacios & 3.5 & 0.9 \\
\hline & Utilidades & 3.6 & 0.7 \\
\hline & Diseño y estilo & 3.6 & 0.9 \\
\hline \multirow{3}{*}{$\begin{array}{c}\text { MANEJO BÁSICO } \\
(1-5)\end{array}$} & Manual & 3.9 & 0.8 \\
\hline & Sesiones tutoradas & 3.8 & 0.7 \\
\hline & Facilidad manejo & 3.6 & 0.6 \\
\hline \multirow{3}{*}{$\begin{array}{l}\text { INTERACCIÓN (1- } \\
5)\end{array}$} & Presencia tutor & 2.4 & 1.1 \\
\hline & Presencia de compañeros & 3.5 & 1.0 \\
\hline & $\begin{array}{l}\text { Sensación de inmersión } \\
\text { conseguida }\end{array}$ & 3.4 & 0.8 \\
\hline \multirow{3}{*}{$\begin{array}{l}\text { CURVA DE } \\
\text { APRENDIZAJE } \\
\text { (Minutos) }\end{array}$} & Desplazamientos & 6.0 & 6.4 \\
\hline & Coger objetos & 6.3 & 5.3 \\
\hline & Personalizar el avatar & 15.3 & 10.2 \\
\hline
\end{tabular}

Del material creado habría que destacar, el mundo virtual adaptado a las reuniones de trabajo (Fig. 2), el manual, el guion de las actividades (Fig. 1). La simulación del entorno virtual 3D fue hecha con OpenSim (http://opensimulator.org/). Para el desarrollo del Proyecto se usó un servidor dedicado a la investigación y desarrollo de nuevos procedimientos para la mejora de la divulgación científica y tecnológica, servidor con 40GB de memoria RAM, 2 CPUs Intel Xeon E5620 2.40GHz 64bits, disco duro 5TB y Ubuntu 10.10, alojado físicamente en el Campus Rabanales de la Universidad de Córdoba. Así el programa Firestorm para Linux fue instalado por el servicio de informática para que los usuarios (estudiantes y profesores) con una clave pudieran acceder al entorno virtual a través su avatar. Este acceso podría continuar usándose. Por otro lado el manual y las sesiones van a ser usados por nuevos usuarios en sucesivas ediciones de la experiencia. En particular se puede citar las experiencias del nuevo Proyecto de Innovación Docente de la convocatoria 16-17 de la Universidad de Córdoba titulado: La aplicación de los mundos inmersivos para la resolución de problemas asociados a casos reales en el mundo de la Ingeniería Agronómica, de Montes y de la Enología, donde el escenario de los mundos inmersivos permitirá interiorizar situaciones comunes del futuro mundo laboral de los egresados tales como fases de una licitación o subastas de productos agroalimentarios. 


\section{UTILIDAD/ANÁLISIS}

La experiencia ha servido para conformar un grupo de trabajo especializado en la adquisición de competencias transversales de comportamiento basadas en mundos virtuales inmersivos. Además, los participantes de este proyecto coinciden en que el impacto sobre los estudiantes ha sido importante en términos de fomento de la creatividad y la exploración de las nuevas tecnologías vinculadas a la gestión de proyectos.

\section{CONCLUSIONES/DISCUSIÓN}

La experiencia educativa ha resultado gratamente estimulante para alumnos y profesores a pesar de disponer de unos medios limitados. Los investigadores participantes esperan continuar la línea de trabajo iniciada y aplicar la plataforma a diferentes disciplinas. En relación a las restricciones de la experiencia y las posibilidades de mejora, hay que señalar que no se ha podido detraer más tiempo de la impartición de los contenidos correspondientes a la asignatura y que sería importante avanzar en el diseño de actividades concretas relacionadas con los contenidos de la asignatura para realizarlas en ambientes inmersivos, explorando la posibilidad que estos ambientes tienen para el entrenamiento de habilidades en situaciones que simulen situaciones y ambientes reales del mundo profesional y laboral.

\section{AgRADECIMIENTOS}

Este trabajo fue financiado parcialmente por la Universidad de Córdoba a través de su convocatoria para Proyectos de Innovación Educativa (Ref. 2015/2/5020).

\section{Bibliografía}

BOURAS, C., \& TSIATSOS, T. "Educational virtual environments: design rationale and architecture". Multimedia Tools and Applications 2006/29(2), 153-173.

UNIVERSIDAD DE CÓRDOBA. 2015. Acuerdo de Consejo de Gobierno, en sesión ordinaria de 27/05/2015, por el que se aprueba el Plan de Innovación Docente del curso 2015/2016 de la Universidad de Córdoba.

BOUCO, 1/6/1015; Anuncio 2015/00198.

Burdea G, Coiffet P. "Virtual Reality Technology". Presence: Teleoperators and virtual environments 2003/12(6), 663-664.

GERALD, S., \& ANTONACCI, D. M. "Virtual world learning spaces: developing a second life operating room simulation". EDUCAUSE Quarterly, 2009/32(1).

LORENZO, C.M., SICILIA, M.A., SÁNCHEZ S. "Studying the effectiveness of multi-user immersive environments for collaborative evaluation tasks". Computers \& Education 2012/59, 1361-1376

MATHERS N., GOKTOGEN A., RANKIN J., ANDERSON M. "Robotic Missionto Mars:Hands-on,mindson,web-basedlearning". Acta Astronautica 2012/80, 124-131

MAMO, M.; NAMUTH-COVERT, D.; GURU, A.; NUGENT, G.; PHILLIPS, L.; SANDALL, L.; KETTLER, T.; MCCALLISTER. D. "Avatars Go to Class: A Virtual Environment Soil Science Activity". Journal of Natural Resources and Life Sciences Education, 2011/40,114-121

OHLAND, M. W., FRILLMAN, S. A., ZHANG, G., MILLER III, T. K.. 'NC State’s Engineering Entrepreneurs Program in the context of US entrepreneurship programs". En Education that works: the National Collegiate Inventors and Innovators Alliance (NCIIA) 8th Annual Meeting, marzo. 2004, 18-20, pp. 155-164.

PALOMO VADILLO, M. T. (2013). Liderazgo y motivación de equipos de trabajo. ESIC Editorial. Madrid, 2013.

SCHMORROW D.D. "Why virtual?” Theoretical Issues in Ergonomics Science 2009/10(3), 279-282.

SUMNERS, C., REIFF, P, WEBER W. "Learning in an immersive digital theater". Advances in Space Research 2008/42, 1848-1854

WILSON, C. "Avatars, virtual reality technology, and the U.S. military: Emerging policy issues". Retrieved October 28, 2010 from. http://www.dtic.mil/cgi-bin/GetTRDoc?Location¹/4U2\&doc $1 / 4$ GetTRDoc.pdf\&AD $1 / 4$ ADA480182. 